\title{
LA COOPERACIÓN TRANSFRONTERIZA EN LA REGIÓN DEL TRIFINIO Y LA DIFUSIÓN DE MODELOS EUROPEOS DE GOBERNANZA DE LAS FRONTERAS EN AMÉRICA LATINA $^{*}$
}

\author{
Cross-border cooperation in the Trifinio region and the transfer of \\ European transborder governance models in Latina America
}

\author{
Filippo Celata ${ }^{* *}$ \\ Raffaella Coletti ${ }^{* * *}$ \\ Venere Stefania Sanna ${ }^{* * * *}$
}

Recibo: 30 de abril de 2013. Aceptado: 20 de octubre de 2013.

\section{RESUMEN}

En la región del Trifinio se concentran algunas de las experiencias más avanzadas de cooperación transfronteriza de América Latina. Dichas experiencias son promovidas por las instituciones europeas y se han inspirado fuertemente en el modelo europeo de gobernanza de las regiones transfronterizas. Al mismo tiempo, este modelo no ha sido simplemente trasladado de la Unión Europea a la América Latina, sino que también se ha adaptado a las condiciones del contexto y a las características específicas de los procesos de reterritorialización e integración macrorregional que tienen lugar en la región y, más

\footnotetext{
* El conocimiento directo de los procesos de integración transfronteriza centroamericana, y en particular el de la Región del Trifinio, ha sido posible gracias a las oportunidades ofrecidas por el Centro Studi di Politica Internazionale (CeSPI), de Roma, en el ámbito de dos de sus iniciativas: el programa Fronteras Abiertas, realizado por dicha entidad en colaboración con el Instituto Italo-Latino Americano y cofinanciado por la Cooperación Italiana, y el proyecto Fomento de la Cohesión Social e Integración Regional Territorial de los municipios fronterizos del Trifinio centroamericano, coordinado por la Mancomunidad Trinacional Fronteriza Río Lempa y cofinanciado por el Programa URB-AL III de la Comisión Europea.

** Universidad de Roma La Sapienza, Departamento Métodos y Modelos para la Economía, el Territorio y las Finanzas, MEMOTEF, Roma, Italia. Correo electrónico: filippo.celata@uniroma1.it

*** Universidad de Roma La Sapienza, Departamento Métodos y Modelos para la Economía, el Territorio y las Finanzas, MEMOTEF. Centro Studi di Politica Internazionale CeSPI, Roma, Italia. Correo electrónico: raffaella. coletti@cespi.it / raffaella.coletti@uniroma1.it

**** Universidad de Roma La Sapienza, Facultad de Arquitectura, Roma, Italia. Correo electrónico: venere.sanna@ uniroma1.it
} 
en general, en América Latina. A la luz del debate relativo a la europeización, el artículo pretende mostrar cómo el potencial y los límites de este tipo de iniciativas pueden ser adecuadamente comprendidos solo a través del balance que se crea, caso por caso, entre contaminación transnacional y adaptación local, y entre la transferencia de modelos y narrativas (que son típicos de la Unión Europea), y la apropiación de estos por parte de los actores locales.

PALABRAS CLAVES: Cooperación transfronteriza, reterritorialización, europeización.

\begin{abstract}
Some of the most advanced cross-border cooperation initiatives in Latin American are to be found in the Trifinio region. These have been promoted by European institutions and have been inspired by the European model of cross-border governance. The model, however, has not simply been transplanted from Europe, but has been adapted to local circumstances and to the specifics of rescaling and macro-regional integration processes in the region, and in Latin America more in general. In the light of academic debates about Europeanization, this article aims to show how both the potential and the limits of such initiatives may only be properly understood in light of a balance between transnational contamination and local adaptation, and between the transfer of models and narratives that are typical of the EU, and their appropriation by local actors.
\end{abstract}

KEY WORDS: Cross-border cooperation, Rescaling, Europeanization.

\title{
1. INTRODUCCIÓN
}

Desde las primeras experiencias espontáneas realizadas en Europa durante la segunda mitad de la década de 1950, la cooperación transfronteriza ha adquirido una relevancia creciente también fuera del territorio europeo. A partir de la década de 1980 este modelo se ha extendido en otras partes del mundo (Perkmann y Sum 2002), y en particular en América Latina, donde la atención a estas prácticas ha aumentado recientemente y donde se han desarrollado algunas de las experiencias más avanzadas de cooperación transfronteriza entre países no europeos.

El objetivo de este artículo es analizar, a la luz de la experiencia de integración transfronteriza desarrollada en la región del Trifinio, en qué medida la difusión de inicia- 
tivas de cooperación transfronteriza en América Latina representa un ejemplo de europeización y de transferencia de políticas (policy transfer) y cuáles son sus consecuencias.

El modelo europeo de cooperación transfronteriza implica, en primer lugar, la existencia -en el nivel macrorregional- de un marco estable de relaciones transnacionales entre los países involucrados y, en segundo lugar, la participación activa de las instancias locales y regionales fronterizas. La cooperación transfronteriza a nivel local representa, entonces, un ejemplo paradigmático de reterritorialización del Estado y de reorganización de su espacialidad política y administrativa (Brenner 2004), ya que requiere y promueve una multiplicación de las escalas geográficas de organización político-administrativa. En efecto, en América Latina las iniciativas de cooperación transfronteriza se promueven, por un lado, en el marco de los procesos de integración regional (Mercosur, Unasur, Comunidad Andina, ALBA, CAFTA-DR, Sistema de la Integración Centroamericana) y territorial (como la Iniciativa para la Integración de la Infraestructura Regional Sudamericana); y por otro lado, gracias a los procesos de descentralización que han permitido una mayor autonomía de los gobiernos subestatales en su accionar internacional (Rhi-Sausi y Conato 2009; Montero y Samuels 2004). Sin embargo, los dos procesos -de integración transnacional y de descentralización- se desarrollan según caminos peculiares y característicos que se reflejan en las razones y resultados de los experimentos de cooperación transfronteriza realizados. Al mismo tiempo, la experiencia del Trifinio puede ofrecer un punto de vista privilegiado para encuadrar el proceso de reterritorialización que se lleva a cabo en la región y sus consecuencias. La región del Trifinio, en efecto, es una zona de frontera entre Guatemala, Honduras y El Salvador, donde, desde hace 20 años, se realiza un proceso de integración a diferentes niveles (local, nacional y supranacional), que de hecho representa una de las experiencias de cooperación transfronteriza más avanzada en la región latinoamericana.

El artículo pretende evaluar hasta qué punto la difusión de prácticas de cooperación transfronteriza consiste en una transferencia de modelos políticos que son típicos de la Unión Europea (UE) y hasta qué punto dichas iniciativas se adaptan a las características específicas del contexto en el que se actúan. ¿Estamos asistiendo a un proceso de europeización de políticas (Featherstone y Radaelli 2003), incluso más allá de las fronteras europeas? ¿Hasta qué punto el "modelo" europeo de cooperación transfronteriza es aplicable en contextos diferentes? ¿Cuáles son, por otro lado, las características específicas de las experiencias que se realizan en el Trifinio?

La estructura del artículo es la siguiente: la próxima sección se centra en la experiencia de cooperación transfronteriza en Europa, con especial énfasis en su relación 
con los procesos de reterritorialización. La tercera sección introduce la experiencia del Plan Trifinio, mientras que la cuarta se centra en el papel desempeñado en ese contexto a nivel local por las mancomunidades locales y por la Mancomunidad Trinacional Fronteriza Río Lempa. La sección siguiente investiga la dinámica de europeización que se lleva a cabo en la región centroamericana, mientras que el sexto párrafo reflexiona sobre la adaptación local del modelo europeo. Finalmente, la última sección muestra algunas conclusiones.

\section{COOPERACIÓN TRANSFRONTERIZA Y RETERRITORIALIZACIÓN EN EUROPA}

La cooperación transfronteriza en Europa puede ser definida como "una colaboración entre autoridades subnacionales más allá de los límites fronterizos nacionales" (Perkmann 2003: 157) ${ }^{1}$.

Las primeras experiencias formales de cooperación transfronteriza entre autoridades sub-estatales en Europa se remontan a la segunda mitad de los años 50, enmarcadas en un panorama general en el que los gobiernos europeos estaban todavía fuertemente centralizados, especialmente en las actividades ligadas a las relaciones exteriores. A partir de los años 80, la cooperación transfronteriza adquirió una relevancia y una difusión siempre en aumento, cuando la Unión Europea empezó a ocuparse explícitamente de este tema (Coletti 2010).

La atención de la Unión Europea en el tema de la cooperación transfronteriza estuvo directamente relacionada con algunos macroprocesos en marcha en el viejo continente y con la vía de la integración. El Acta Única Europea (AUE), de 1986, llevó, por un lado, a la creación del Mercado Común en 1993, con la libre circulación de bienes, servicios, personas y capitales dentro del territorio comunitario. Por otro lado, la Unión Europea reconoció en ese documento la importancia de crear políticas públicas a escala continental orientadas a paliar desequilibrios regionales y sociales existentes entre las diferentes regiones europeas.

De esta forma, las políticas de cooperación transfronteriza adquieren una nueva relevancia, ya que abordan el tema de los desequilibrios regionales y actúan precisamente en las áreas más afectadas por el impacto del mercado único. En relación al contexto ex-

1 Para un análisis exhaustivo de las características y de la difusión de la cooperación transfronteriza en Europa, véase Coletti 2010. 
terno, la caída del muro de Berlín y la desintegración de la Unión Soviética, modificaron el panorama que circundaba a la Unión Europea, obligándola a poner también particular atención a las fronteras externas.

Debido a esta atención prestada, la cooperación transfronteriza se ha convertido desde la década de 1990 en un tema central de política pública regional, y muchos actores locales han recibido apoyo financiero para realizar actividades de cooperación. Como consecuencia de ello, las prácticas de cooperación se han difundido por toda Europa, incluso en aquellos territorios que espontáneamente no las habían experimentado. De hecho, la difusión de las políticas de cooperación transfronteriza ha seguido un camino local-central-local que puede considerarse típico de cualquier política pública (Uitermark 2005): en particular, las políticas llevadas a cabo por actores centrales (por ejemplo, Estados nacionales o Unión Europea) derivan de procesos y experimentos locales; estas prácticas se exportan al nivel central, y luego, de nuevo pueden ser distribuidas a nivel local en un conjunto más amplio de territorios. La cooperación transfronteriza, que surgió como práctica espontánea a final de la Segunda Guerra Mundial, se ha convertido en una importante política europea, y como tal ha sido redistribuida en toda la Unión. Sin embargo, la propagación desde el nivel central hacia el local, produjo un debilitamiento general de las experiencias de cooperación transfronteriza, tanto en términos de estandarización de las prácticas, que no siempre han garantizado respuestas adecuadas a problemáticas específicas, como en términos de voluntad política de las autoridades locales y regionales a cooperar, a menudo subordinadas al deseo de acceder a los fondos puestos a disposición por la Unión Europea para la cooperación territorial.

En todo caso, las razones de base para la difusión de la cooperación transfronteriza se encuentran en las múltiples dimensiones que estas prácticas han estimulado y sobre las que se ha actuado (Coletti 2010). Por ejemplo, las prácticas de cooperación transfronteriza han permitido que las autoridades locales y regionales europeas afronten de forma conjunta problemáticas comunes, como la gestión natural de un mismo ecosistema o la reconversión de zonas industriales transfronterizas.

Otro elemento clave, al que las prácticas de la cooperación transfronteriza han contribuido, es el intercambio de prácticas entre actores del territorio (instancias subestatales y sociedad civil) que pertenecen a diferentes Estados: intercambio que ha generado un proceso de integración desde abajo, convirtiéndose en un pilar de la integración y del desarrollo de la Unión Europea. 
Finalmente, las prácticas de cooperación transfronteriza se han convertido en un importante instrumento en el ámbito del desarrollo económico: las áreas de frontera suelen ser periféricas respecto a sus centros nacionales y a veces se ven afectadas negativamente por las políticas de los propios Estados. En relación a esto, en el caso europeo, las prácticas de cooperación transfronteriza han ofrecido a los territorios la posibilidad de crear nuevos centros a escala transnacional, complementando muchas funciones localizadas tradicionalmente en el centro de los respectivos países.

Si bien estos son solo algunos de los resultados de las prácticas de cooperación transfronteriza, el principal valor añadido de las políticas en este ámbito se encuentra en su dimensión inmaterial y simbólica (Celata y Coletti 2011). La cooperación transfronteriza ha apoyado el proceso de integración no solo de forma concreta, a través de la difusión de las iniciativas de cooperación entre territorios, sino también a nivel simbólico, por medio de la promoción de una imagen del territorio europeo interconectado, fluido y con fronteras abiertas. Desde este punto de vista, las prácticas y políticas de cooperación transfronteriza han contribuido a reforzar la idea de una Europa unida, que inspira y guía el proceso concreto de integración (Coletti 2010).

El valor simbólico de la cooperación transfronteriza se manifiesta entonces, en todas la dimensiones que caracterizan a la reterritorialización (rescaling) europea, o sea, la reconfiguración de la jerarquía del espacio geográfico fundado sobre la base de las estructuras sociales e institucionales de un sistema dado (Brenner 2004). La superación de las fronteras nacionales que presupone la cooperación transfronteriza contribuye a la reterritorialización de la Unión Europea, ya que simboliza la superación de la tradicional subdivisión del sistema westfaliano, en la que se transforma a Europa de una simple suma de Estados más o menos descentralizados en un sistema político-geográfico muy complejo, poroso y multinivel.

Con el fin de estimular el proceso de creación de la territorialidad europea, la Unión Europea trata de superar las tradicionales divisiones nacionales conocidas en el imaginario colectivo. La gestión de los límites fronterizos y el tema de la cooperación transfronteriza son claros ejemplos de una gobernanza multinivel (Hooge y Marks 2001): a nivel vertical, puesto que existe una división de funciones entre los diferentes niveles institucionales, y a nivel horizontal, debido a la participación de distintos actores territoriales públicos y privados. Con base en diferentes ámbitos -locales, nacionales y supranacionales-, la Unión Europea contribuye a la identificación de un modelo y, por lo tanto, de un territorio europeo, que no es simplemente la suma de los diferentes Estados nacionales. 
Además de esta relativización de escalas, la cooperación transfronteriza promueve un proceso similar que se puede definir de rebordering, o sea, un proceso donde las fronteras adquieren un nuevo significado a la luz de los procesos de integración que ocurren a las diferentes escalas.

En distintas partes del mundo se experimenta un proceso similar de reterritorialización, en el marco del cual la experiencia europea simboliza un papel clave, como se analizará en los párrafos siguientes en relación con el caso del Trifinio.

\section{LA INTEGRACIÓN TRANSFRONTERIZA EN LA REGIÓN DEL TRIFINIO}

Como ya ha sido mencionado en la introducción, también América Latina ha experimentado, en los últimos años, cierta difusión de iniciativas de cooperación transfronteriza a nivel nacional y local. Una de las experiencias de mayor interés es, sin duda, el caso de la región del Trifinio, la zona de frontera donde Guatemala, Honduras y El Salvador convergen alrededor del macizo montañoso de Montecristo².

El Trifinio puede considerarse como una región homogénea, en primer lugar, por sus dimensiones sociales, económicas y productivas (Sanna 2011); seguidamente, desde el punto de vista geofísico, por constituir el área de origen de los sistemas hídricos más importantes de Centroamérica (ríos Lempa, Motagua y Ulúa) y por sus características naturales y biológicas homogéneas. Además, desde el punto de vista geoestratégico, el Trifinio constituye un área significativa por sus rasgos a escala regional: en un radio de 250 kilómetros desde el punto Trifinio se localiza el 60 por ciento de la población de los cinco países centroamericanos, se concentra el 75 por ciento de la red vial centroamericana y se llega a tres capitales, dos complejos portuarios en el Atlántico y cuatro complejos portuarios en el Pacífico.

La suma de su relevancia estratégica y de su propia naturaleza transfronteriza configura al Trifinio como un área de natural experimentación de prácticas y políticas

2 El macizo montañoso de Montecristo es una zona ecológica de bosque nuboso declarada Reserva Internacional de La Fraternidad, en 1987 y desde 2011, patrimonio de la humanidad por la Unesco. El Trifinio comprende aproximadamente 7.541 kilómetros cuadrados, de los cuales 44,7 por ciento corresponde a Guatemala, 40 por ciento a Honduras y 15,3 por ciento a El Salvador. La región representa el 13 por ciento de la superficie total de los tres países, acoge el 3 por ciento de sus habitantes totales y comprende 45 municipios fronterizos: 15 guatemaltecos, 22 hondureños y 8 salvadoreños. 
de cooperación transfronteriza y gobernanza local. Sin embargo, estas características no son suficientes para establecer un proceso de integración transfronteriza. Para tal fin, es necesario que exista la voluntad política -tanto a nivel local como a nivel de los Estados involucrados-, necesaria para avanzar con un proyecto de cooperación.

\section{Figura No 1}

Los municipios de la región Trifinio

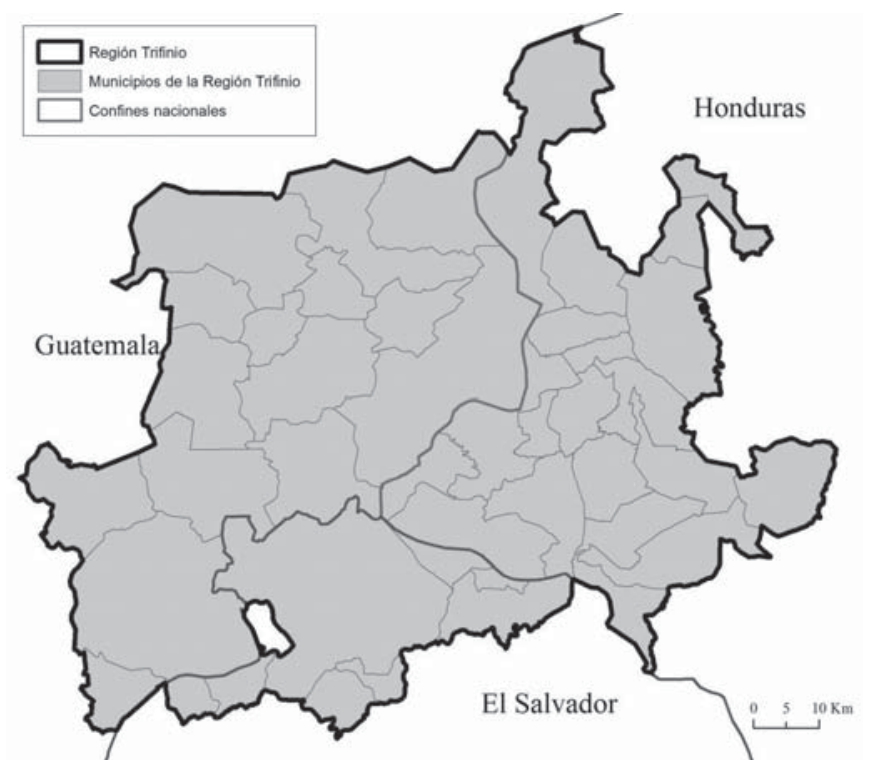

Fuente: Elaboración propia

La región del Trifinio es parte de una serie de procesos políticos especialmente activos hacia la integración de Centroamérica. En primer lugar, a escala supranacional, en las últimas décadas los países del istmo centroamericano han implementado varios instrumentos y políticas económicas y comerciales, para avanzar con el proceso de integración regional. Para tal efecto, se desarrolló una compacta red institucional para apoyar el proceso de integración que actualmente es liderado por el Sistema de la Integración Centroamericana (SICA), creado en 1991, por los Estados de Costa Rica, El Salvador, Guatemala, Honduras, Nicaragua y Panamá3 , con el fin de realizar la integración de Centroamérica.

3 El SICA fue constituido, el 13 de diciembre de 1991, mediante la suscripción del Protocolo de Tegucigalpa, y entró en funcionamiento formalmente el 1 de febrero de 1993. Posteriormente se adhirió Belice como miembro pleno y la República Dominicana como Estado asociado. Precedentemente, en 1960, Guatemala, El Salvador, 
En lo que se refiere a escala nacional, a lo largo de la historia, El Salvador, Honduras y Guatemala, tal como los otros Estados del istmo, estuvieron en guerra civil hasta el siglo XX, "razón por la cual la reconstrucción democrática y la modernización política han ido de la mano con el diseño de nuevos mecanismos de paz y gobernanza regional" (Rhi-Sausi y Oddone 2009: 50). Más allá del proceso de integración regional, entre los tres Estados existe un listado de acuerdos plurinacionales de colaboración e intercambio.

Por último, llegando al nivel local (y tomando en cuenta también la inexistencia de niveles de gobierno intermedios), es importante mencionar el renovado papel de los municipios. Actualmente en estos países, los gobiernos locales ostentan amplias competencias (por lo menos desde un punto de vista formal) en sectores claves para el desarrollo económico y, de acuerdo al proceso de descentralización en curso, en muchos países centroamericanos se reconoce cada vez mayor autonomía municipal.

Bajo este marco institucional, en la región "se está viviendo desde hace veinte años un proceso de integración transfronteriza sin precedentes en América Latina" (Conato 2009: 129).

El primer experimento relevante de efectiva cooperación institucionalizada tuvo lugar, en 1986, cuando los gobiernos de Guatemala, Honduras y El Salvador, la Secretaría General de la Organización de los Estados Americanos (OEA) y el Instituto Interamericano de Cooperación para la Agricultura (IICA), suscribieron un acuerdo de cooperación con el objetivo de formular el Plan de Desarrollo Integral en la Región Fronteriza, denominado "Plan Trifinio". Finalmente, el Plan Trifinio nació en 1988 y fue actualizado en 1992 y en 2004. En 1997, se firmó el Tratado entre las Repúblicas de El Salvador, Guatemala y Honduras para la Ejecución del Plan Trifinio.

De hecho, el proceso de acercamiento entre los tres gobiernos nacionales se basó en la preocupación por la conservación forestal: la defensa del bosque nebuloso y de la biodiversidad del macizo de Montecristo. Sin embargo, además del interés mostrado por el manejo compartido de los recursos forestales de la Reserva de la Biósfera La Fraternidad"

Hay una motivación profunda para el Plan Trifinio, más allá del interés inicial en el área de conservación y en el mismo Plan de Desarrollo Integral en la Región Fronte-

Honduras y Nicaragua suscribieron el Tratado General de Integración Económica Centroamericana, uno de los procesos de integración más antiguos de Latinoamérica, al que Costa Rica se adhirió dos años después, iniciando así el denominado Mercado Común Centroamericano (MCCA). 
riza, de encontrar una alternativa más viable, efectiva y de resultados importantes a corto plazo, de esfuerzos de integración plurinacional (Organización de los Estados Americanos e Instituto Interamericano de Cooperación para la Agricultura 1988).

En términos más generales, como ha sido declarado por el mismo SICA, el objetivo fundamental del plan es contribuir a la integración centroamericana, mediante una acción conjunta de El Salvador, Guatemala y Honduras, que tienda al desarrollo integral, armónico y equilibrado de la región fronteriza de los tres países.

El proceso de creación de la región transfronteriza Trifinio parece interesante por distintas razones. En primer lugar, el tratado define el ámbito geográfico de la región del Trifinio y el espacio de acción compartida constituido por la misma -mediante la concreta institucionalización del escenario territorial de la cooperación trinacional-, declarándola como "una unidad indivisible, en la que sólo una acción conjunta y coordinada de los tres países podrá dar solución satisfactoria a los problemas de sus poblaciones y al manejo sostenible de sus recursos naturales" (Comisión Trinacional del Plan Trifinio 1998: Cap. I, art. 3). Consecuentemente, la región transfronteriza es "naturalizada" a nivel político y a escala regional. Se activa de esta forma un proceso de rescaling en el que nuevos límites de la región transfronteriza superan las tradicionales particiones nacionales impuestas por el sistema westfaliano.

En segundo lugar, la experiencia del Trifinio se destaca por el modelo de gobernanza utilizado. El tratado institucionaliza la máxima autoridad trinacional: la Comisión Trinacional del Plan Trifinio (CTPT) (Figura 2), que es el ente encargado de tutelar la ejecución del Plan Trifinio y de su permanente actualización. La comisión es responsable de impulsar "la cooperación transfronteriza, la gestión conjunta de financiamiento, la inversión compartida de los tres países y el manejo integrado de los recursos naturales" (Task Team on South-South Cooperation 2011: 9).

Como se muestra en el organigrama (Figura No 2), la estructura organizativa de la comisión revela una dinámica de devolución "hacia abajo", donde los gobiernos nacionales delegan parte de sus competencias a un organismo tripartito que tiene su propio fundamento territorial en un área transfronteriza definida por tratado. 
Volumen XIII / No 2 / julio - diciembre 2013 / pp. 165-189

Figura No 2

El Organigrama de la Comisión Trinacional del Plan Trifinio (CTPT)

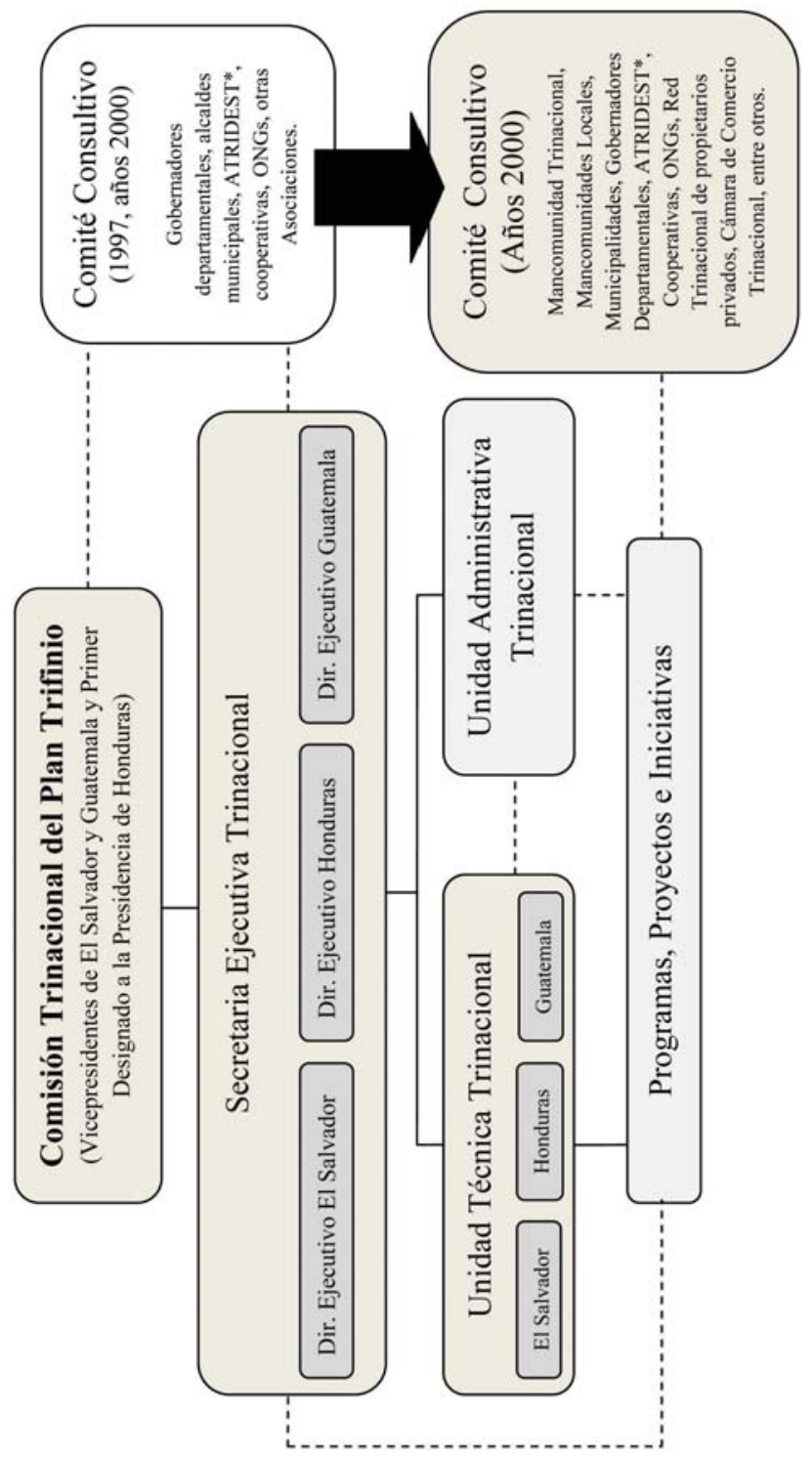

Fuente: Elaboración propia con información de Comisión Trinacional del Plan Trifinio 2004. 


\section{COOPERACIÓN TRANSFRONTERIZA A NIVEL LOCAL EN LA REGIÓN TRIFINIO}

Paralelamente al proceso de cooperación para el establecimiento de la región del Trifinio a nivel intergubernamental, a nivel local ya desde hace décadas se han venido desarrollado entre algunos municipios de Guatemala, Honduras y El Salvador, procesos espontáneos de cooperación entre fronteras; integración que se ha basado en una dinámica y sistemática relación comercial, que se explica en parte, por la marginalidad y falta de fuerte relación de estos territorios con los centros poblacionales de los respectivos países y, asimismo, por los procesos recientes de generación e interconexión entre estos municipios (Artiaga 2003).

En efecto, las instancias locales jugaron un papel relevante también en el marco de la creación del Plan Trifinio. De hecho, el plan surgió de forma consensuada, y a partir de un proceso participativo entre los líderes locales y los gobiernos de los tres países, con el objetivo específico de lograr el desarrollo integral equilibrado de la región, mediante la acción conjunta y coordinada a múltiples escalas, nacionales y locales, en el marco de la cooperación transfronteriza y de la integración centroamericana.

Los gobiernos locales han mantenido un papel central en el marco del Plan Trifinio durante todo su curso y desarrollo, tanto que en 2004, con ocasión de su actualización se realizó un nuevo proceso participativo, que involucró a actores de todas las escalas administrativas.

Sin embargo, el papel de los actores locales no se ha limitado a una forma de participación en los procesos en curso a nivel central. Los gobiernos locales han desarrollado actividades directas de cooperación intermunicipal. De hecho, como iniciativa de los gobiernos municipales de los tres países (y en algunos casos con el apoyo técnico y económico de la Comisión Trinacional del Plan Trifinio), las municipalidades han venido organizándose en uniones de municipios para generar economías de escala y resolver problemas comunes.

Solo para dar un ejemplo, entre el 2003 y 2006 en el Trifinio se crearon seis asociaciones de carácter local-nacional y paralelamente se inició un proceso de legalización de una mancomunidad trinacional de gobiernos locales: la Mancomunidad Trinacional Fronteriza Río Lempa (MTFRL) ${ }^{4}$ que debe su nombre a la cuenca hidrográfica trinacio-

4 Reconocida jurídicamente en Guatemala, por el Ministerio de Gobernación (2007), en Honduras por la Secretaría de Gobernación y Justicia (2009) y en la República de El Salvador según publicación en el Diario Oficial (2011). 
nal. Como se puede observar mediante la comparación del mapa de las mancomunidades locales (Figura No 3 ) con el mapa de los municipios que componen la región del Trifinio (Figura $\mathrm{N}^{\circ} 1$ ), hay una diferencia entre la cobertura geográfica del área ocupada por las mancomunidades (locales y Mancomunidad Trinacional Fronteriza Río Lempa) con el área de la región definida por el Plan Trifinio.

\section{Figura No 3}

Las mancomunidades locales y la Mancomunidad Trinacional Fronteriza Río Lempa

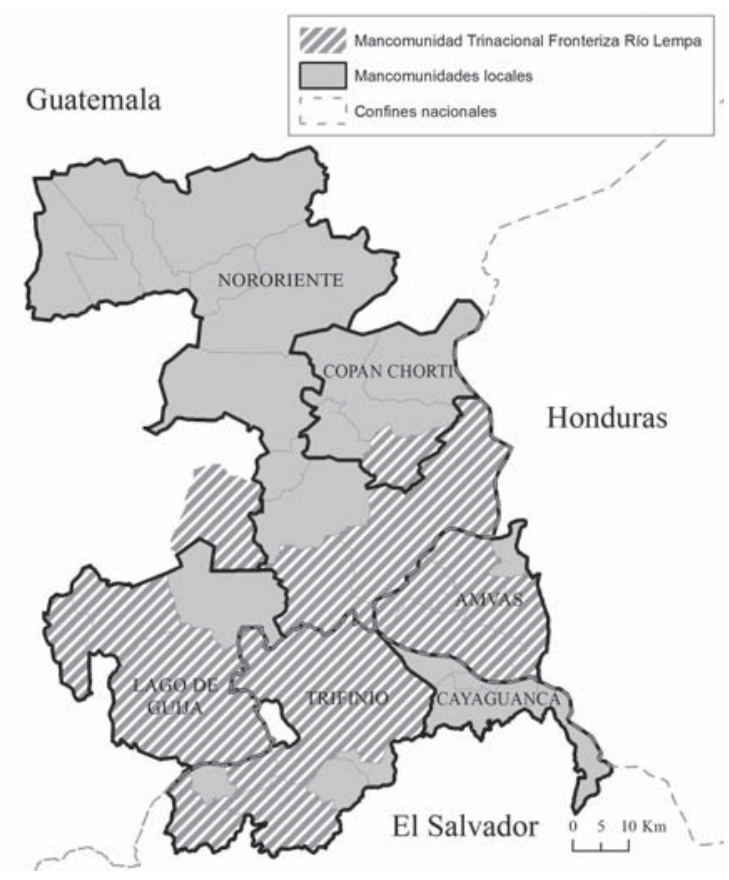

Fuente: Elaboración propia

La Mancomunidad Trinacional Fronteriza Río Lempa, integrada por 12 municipios de los tres países (seis municipios guatemaltecos, cuatro hondureños y dos salvadoreños, cada uno de los cuales pertenece a alguna mancomunidad local-nacional), a partir del 2007 se convirtió en una institución pública local autónoma, laboratorio de cooperación transfronteriza con el objetivo de "garantizar la plena, integrada y efectiva participación de los gobiernos municipales en la gestión conjunta del desarrollo de la región... a través de la formulación y ejecución de políticas públicas, planes, programas 
y proyectos municipales, intermunicipales y subregionales" (Mancomunidad Trinacional Fronteriza Rio Lempa 2008).

Varios autores (Artiaga 2003; Cardona 2009; Rhi-Sausi, Conato, Oddone y Salerno 2011) señalan que la Mancomunidad Trinacional nació específicamente para ofrecer una alternativa concreta a la débil participación de gobiernos locales en las actividades del Plan Trifinio y a la falta de coordinación entre mancomunidades fronterizas de los tres países. Sin embargo, vale la pena resaltar que, en el marco de una integración multinivel, el nivel local, por su misma naturaleza, es capaz de abordar problemas específicos que no pueden ser afrontados por el nivel nacional. No por casualidad, la Mancomunidad Trinacional Río Lempa llevó a cabo un proceso de planeación estratégica territorial trinacional (PET 1998-2003), "estableciendo los primeros pasos para articular a los actores locales en relación a una agenda multi-país, multinivel y pluri-actoral" (Task Team on South-South Cooperation 2011: 11). Sustancialmente, con el paso del tiempo,

se formalizó una alianza estratégica con otras mancomunidades de la región, generándose así la práctica de la cooperación entre un municipio y otro, tomando como punto de partida una agenda común más amplia e integral que la establecida por la Comisión Trinacional del Plan Trifinio, con objetivos planteados de acuerdo al diagnóstico local y a los objetivos estratégicos (Task Team on South-South Cooperation 2011: 11).

Actualmente, las mancomunidades han sustituido la participación individual de los alcaldes en el Comité Consultivo del Plan Trifinio (Figura No 2). De esta manera, las mancomunidades, y en particular la Mancomunidad Trinacional, se convierten, por un lado, en interlocutores verticales en el proceso de cooperación entre Estados, proceso que ha determinado una redefinición de la región del Trifinio; y, por otro lado, enriquecen los procesos transfronterizos con nuevos impulsos para la cooperación mediante alianzas estratégicas de tipo horizontal.

La región del Trifinio se presenta, entonces, como una entidad compleja, multinivel, pluriactoral y con una geometría variable, sede y punto de coordinación de políticas y de obtención de fondos de financiación para el manejo de recursos compartidos en el territorio.

Se señala también que la estructura del Trifinio permite una gestión integrada de los fondos de financiación para el desarrollo de la zona, provenientes de diversos donantes internacionales; de esta manera no solo se trata de evitar la duplicación y la dispersión excesiva de fondos, sino también de alcanzar una masa crítica de recursos para la gestión 
unitaria de los problemas de la zona. Aunque la débil capacidad institucional de los municipios y la falta de correspondencia entre sus competencias teóricas y los recursos disponibles (provenientes del nivel central), como se mencionara anteriormente, constituye una fragilidad crucial de los municipios.

Sin embargo, el papel de los municipios y las mancomunidades no es únicamente importante para ofrecer una respuesta conjunta a los desafíos sociales y económicos de la región, sino también a nivel simbólico: la compleja geografía de las mancomunidades contribuye a presentar la imagen de una región de múltiples escalas, fluida y sin fronteras. Las oportunidades concretas de cooperación entre municipios que las mancomunidades ofrecen, junto con el significado simbólico que esta cooperación tiene, contribuyen al mantenimiento de la paz, al proceso de integración regional y al inicio de un proceso de desarrollo dinámico compartido.

\section{V. ¿LA COOPERACIÓN TRANSFRONTERIZA EN LA REGIÓN DEL TRIFINIO COMO EJEMPLO DE EUROPEIZACIÓN?}

Por lo que se ha dicho hasta ahora, es evidente que el caso del Trifinio presenta una serie de similitudes con la experiencia europea, tanto en lo que concierne al proceso de integración en curso a nivel supranacional, como con respecto al activismo de los gobiernos locales en los procesos de cooperación transfronteriza. Las similitudes son discernibles también en las motivaciones que han animado al proceso de integración y en sus consecuencias. En términos de motivaciones, a semejanza de lo ocurrido en Europa, la integración transfronteriza surge, por un lado, por motivaciones concretas de gestión conjunta, y por otro lado, asume una relevancia política que simboliza la voluntad de los actores involucrados por potenciar la cooperación y la integración, y para crear de forma concreta una plataforma institucional donde dicha cooperación pueda desarrollarse. En lo que se refiere a las consecuencias, la cooperación transfronteriza ha determinado la definición de un nuevo nivel de gobernanza en el área del Trifinio, nivel que va más allá de las fronteras nacionales, de manera similar a lo que ocurrió en Europa.

En este contexto, la pregunta de análisis que se intenta explorar es la siguiente: ¿en qué medida la experiencia del Trifinio puede ser reinterpretada como un caso de europeización externa; es decir, de distribución de formas de organización política y de gobernanza típicas de Europa más allá de sus límites territoriales (Olsen 2002)? ¿Cuáles son, eventualmente, las consecuencias de esta transferencia de políticas (policy transfer)? 
¿Cuáles son, en su lugar, las características específicas del Trifinio en comparación con experiencias europeas similares?

Según Featherstone y Radaelli, la europeización consiste en:

procesos de construcción, difusión e institucionalización de reglas formales e informales, procedimientos, paradigmas de policy, estilos o formas de hacer las cosas, y de valores y normas compartidas que inicialmente se definen y consolidan en el proceso político de la UE para ser incorporados posteriormente en la lógica del discurso, las estructuras políticas y las políticas públicas internas (estatales y subestatales) (2003: 333).

La europeización se aplica también a los territorios externos de la Unión Europea, a través de una voluntad directa de la misma Unión, o por mecanismos indirectos basados en la adopción de una determinada manera de hacer las cosas, o incluso mediante mecanismos de imitación (Schimmelfennig 2010).

En el Trifinio hay por lo menos dos diferentes tipos de europeización: uno que se refiere a la transferencia de modalidades institucionales concretas, reglamentarias y organizativas; $y$ otro que hace referencia a modos de hacer y pensar, y a narrativas y representaciones. Ambos aspectos, en el caso del Trifinio, se realizan en escalas diferentes: la transferencia no se expresa solamente a nivel supranacional, sino igualmente involucra niveles administrativos subyacentes y las relaciones entre ellos.

Un primer nivel de europeización es visible a escala supranacional. En efecto, en lo que se refiere al proceso de integración centroamericano, "sus periodos históricos guardan ciertas similitudes con las reformas que se han dado en Europa para el fortalecimiento de la Unión económica y política" (Guerrero 2004: 995). Aunque las condiciones políticas, económicas, sociales y culturales eran muy diferentes, el acercamiento al proceso de integración ha experimentado pasos similares, desde la creación de una unión aduanera y económica (MMCA), hacia una integración más compleja en el marco del SICA.

En lo que se refiere a los niveles estatal e intergubernamental, los países que componen el Trifinio han suscrito diferentes acuerdos, entre otros: (i) el Tratado de Libre Comercio entre Honduras, El Salvador, Guatemala y México: área del Triángulo Norte (2000) y (ii) el Convenio de Creación de Visa Centroamericana para la libre movilidad de extranjeros entre las repúblicas de El Salvador, Guatemala, Honduras y Nicaragua denominado CA-4 (2004). Es relevante subrayar que ambos acuerdos replican lo ocurrido en Europa mediante la creación del Mercado Único (1993) y el acuerdo de Schengen 
$(1995)^{5}$. En ambos casos, las zonas fronterizas han experimentado procesos de transformación profunda y también adquieren un papel simbólico importante, convirtiéndose en objeto de creciente atención. La cooperación transfronteriza es una herramienta factible para la gestión de estas transformaciones y la Unión Europea no solo es una significativa fuente de inspiración en este sentido, sino también representa una importante fuente de financiación de dichas iniciativas. En efecto, cabe señalar que la Unión Europea ha impulsado el proceso en sus diferentes pasos mediante un explícito apoyo financiero al Plan Trifinio: ya en 1986, para la formulación de los estudios destinados a la elaboración del Plan Trifinio (1988), la Comunidad Económica Europea concedió tal apoyo. Posteriormente, tuvo un papel especialmente activo en el marco de una de las experiencias de cooperación conjunta más importantes en la región que fue el Proyecto Piloto Trifinio, ejecutado entre 1992 y 1999, con una inversión del 75 por ciento proveniente de la Unión Europea (Comisión Trinacional del Plan Trifinio 1999).

La influencia del modelo europeo es evidente también en las narrativas y representaciones utilizadas; no es una coincidencia que, entre otros, los objetivos del Plan Trifinio de "lograr el desarrollo integral, armónico y equilibrado de la región fronteriza de los tres países" (OEA e IICA 1988: 22) son tan similares a los acordados en el marco del tratado constitutivo de la Comunidad Europea del 1957 de "promover... un desarrollo armonioso, equilibrado y sostenible de las actividades económicas en el conjunto de la Comunidad" (Comunidad Europea 1957: Artículo 2).

Finalmente, a otra escala, un proceso de europeización se evidencia a nivel local, y consiste en una contaminación entre la experiencia europea de cooperación transfronteriza y las características específicas de las mancomunidades. Esta contaminación ha sido particularmente fuerte y significativa en los últimos años y ha llevado, por ejemplo, a la creación de la Mancomunidad Trinacional Fronteriza, ya que este tipo de plataforma nació en Europa a finales de la década de 1950 y desde entonces se ha convertido en un ejemplo paradigmático.

Posteriormente, la europeización se realiza, de hecho, mediante el diálogo entre mancomunidades centroamericanas y una serie de instancias locales europeas, contrapartes en los procesos de cooperación descentralizada. El tema de la cooperación transfronteriza ha adquirido una importancia creciente en el marco de la cooperación descentralizada (entre gobiernos locales) entre la Unión Europea y América Latina. Este

$5 \quad$ El acuerdo de Schengen permite suprimir los controles en las fronteras interiores entre los Estados signatarios y crear una única frontera exterior donde se efectúan los controles de entrada en el espacio de Schengen con arreglo a procedimientos idénticos. 
dio como resultado, por ejemplo, iniciativas concretas de cooperación e intercambio de prácticas, como el Programa Fronteras Abiertas, cofinanciado por el gobierno italiano y dedicado expresamente a sostener la cooperación transfronteriza a nivel local en América Latina (Rhi-Sausi y Conato 2009; Marteles 2010). Esta contaminación se expresa asimismo en la creciente atención al tema de la cooperación transfronteriza en las publicaciones del Observatorio sobre la cooperación descentralizada UE-AL (www.observocd.org). Además, el programa Urb-Al III, financiado por la Unión Europea, tiene como objetivo apoyar la cooperación y el intercambio de experiencias entre instancias subestatales europeas y latinoamericanas, incluso en materia de cooperación transfronteriza. Un ejemplo, en el caso específico de la Mancomunidad Trinacional Río Lempa, es el proyecto "Fomento de la Cohesión Social e Integración Regional Territorial de los municipios fronterizos del Trifinio Centroamericano". La europeización en el área, a través del programa Urb-Al, se lleva a cabo en dos niveles diferentes: por un lado, a través de la provisión de fondos destinados a prioridades temáticas específicas, el programa influencia y orienta la cooperación transfronteriza; y por otro lado, favorece la transferencia de conocimientos y modos de cooperación de las autoridades subestatales de Europa hacia sus contrapartes latinoamericanas.

\section{LA ADAPTACIÓN LOCAL DE UN MODELO EUROPEO}

Los estudios críticos sobre la europeización han hecho hincapié en la complejidad de las redes de actores horizontales y verticales a través de las cuales los modelos de políticas se mueven entre los territorios. En un marco de transferencia de modelos políticos tan complejo, el papel de algunos actores específicos es crucial. Entre la variedad de actores que desempeñan un papel crucial en la transferencia de modelos políticos, los "empresarios políticos" (Dolowitz y Marsh 1996: 345) y los técnicos son particularmente importantes en la transferencia de modelos políticos (Benson y Jordan 2011:369; Prince 2010). Lo que emerge de estas investigaciones, sin embargo, es que "hay que evitar la tentación de entender la transferencia de modelos políticos a través de una sencilla metáfora de importación y exportación" (McCann y Ward 2012: 327): los modelos no solo se transfieren de un lugar a otro, pero siempre se adaptan a las circunstancias locales.

Según Peck y Theodore, por ejemplo, las transferencias son siempre "selectivas y multilaterales" (2001: 449). McCann y Ward definen estas adaptaciones como mutaciones (2012: 329). 
Las políticas móviles rara vez viajan como paquetes completos, sino que se mueven en partes y piezas -como discursos selectivos, ideas incipientes y modelos de síntesis- y por lo tanto llegan no como réplicas, sino como políticas ya en transformación (Peck y Theodore 2010: 170).

Además, estas mutaciones y adaptaciones son cruciales para el éxito o fracaso de cualquier transferencia de políticas: la exportación de modelos de cooperación transfronteriza en sí misma no implica que las experiencias que han funcionado en Europa se puedan replicar por igual en contextos diferentes. A menudo, la replicación de modelos de política no produce el resultado esperado y una buena práctica en algún lugar puede convertirse en una mala práctica en otro.

La experiencia del Trifinio se diferencia de la experiencia europea en al menos cuatro aspectos fundamentales: (i) el más débil contexto de descentralización y de subsidiariedad; (ii) el más frágil contexto de integración supranacional en el cual las relaciones transfronterizas están enmarcadas; (iii) la naturaleza de los fondos de financiación disponibles para la cooperación transfronteriza; y (iv) el modelo de gobernanza multinivel utilizado.

Con respecto al contexto de descentralización y de subsidiariedad, las tendencias de descentralización en curso a escala centroamericana han determinado un aumento de responsabilidades y competencias de los gobiernos locales. Sin embargo, muchas críticas permanecen. Desde el punto de vista institucional, en la mayoría de los casos (i) aún existe una desarticulación entre agenda de descentralización y reforma del Estado; (ii) persisten vacíos de funciones y, como consecuencia, las municipalidades tienen un rol poco participativo (sobre todo las más pequeñas, que disponen de menos recursos financieros y humanos); y (iii) se nota una marginalidad generalizada de los gobiernos locales en los procesos nacionales, aunque hay casos puntuales exitosos entre grandes municipalidades (Sanna 2011). El avance en el proceso de descentralización es una condición necesaria para garantizar la sostenibilidad de la experiencia de cooperación en el Trifinio, debido al papel clave jugado por los municipios en este contexto.

En cuanto a la integración supranacional, la integración centroamericana, según varios autores, es aún "una realidad confusa" caracterizada por problemas tradicionales como "la dispersión institucional y las dudas sobre la voluntad política de los Estados centroamericanos de convertir el proceso regional en un marco de políticas de desarrollo" (Caldentey y Romero 2010: 223). Consecuentemente, "el único ámbito en el que se podría hablar de políticas verdaderamente comunitarias es la unión aduanera” 
(Caldentey y Romero 2010: 225). El proceso de integración centroamericana es, sin duda, muy débil en comparación con el nivel de integración alcanzado por la Unión Europea y esto es evidente observando la falta de mecanismos claros y consolidados de gestión en las relaciones entre niveles de los gobiernos o la debilidad del mismo SICA como actor líder del proceso de integración.

Sin embargo, el SICA ofrece un marco de referencia y una plataforma de diálogo para instancias de diferente naturaleza y escala, para actividades de cooperación. Como en el caso de la descentralización, el avance en el proceso de integración podrá favorecer el desarrollo de la cooperación transfronteriza en el Trifinio.

Además, en el ámbito de aplicación de la disponibilidad de fondos se manifiestan las principales diferencias entre el caso del Trifinio y las experiencias europeas similares. En efecto, mientras que los fondos europeos para la cooperación transfronteriza son de fuente interna (Comisión Europea, Estados nacionales y gobiernos locales), en el Trifinio los recursos provienen principalmente de la cooperación internacional. Esto genera un marco diferente donde la misma cooperación se lleva a cabo. En Europa, la cooperación transfronteriza es vista como un componente de un proceso sistémico de integración en el que las diferentes iniciativas de cooperación se enmarcan en ejes y recursos establecidos de forma consensuada entre diferentes niveles institucionales y actores, para perseguir fines institucionales propios de la Unión. En el caso del Trifinio, a diferencia de lo anterior, el proceso de integración en diferentes escalas (SICA, Trifinio y Mancomunidad Trinacional), se activa e inspira no solo por los recursos y finalidades locales, sino también por los impulsos (ideológicos y económicos) de diferentes donantes internacionales.

En este sentido, la misma naturaleza de la experiencia y los problemas son diferentes. Las instituciones del Trifinio representan los puntos de coordinación y armonización no solo entre las diferentes escalas en las que la cooperación se desarrolla, sino también entre diferentes impulsos provenientes desde el exterior.

Finalmente, una última diferencia es evidente en el modelo de gobernanza que se deriva de la creación de la Comisión Trinacional del Trifinio que no tiene parangón alguno en Europa, donde se establecen exclusivamente instituciones transnacionales de cooperación transfronteriza a nivel local. Entonces este modelo de gobernanza representa una tipología de manejo de la cooperación transfronteriza única y peculiar, propio de la experiencia centroamericana. 


\section{CONCLUSIONES}

Este artículo tenía el objetivo de reflexionar sobre la experiencia de la región del Trifinio a la luz de la difusión de las prácticas de cooperación transfronteriza y de la experiencia adquirida por la Unión Europea en este ámbito.

La experiencia de la región del Trifinio se puede interpretar como una práctica de europeización externa, en la medida en que la Unión Europea ha sido un ejemplo y modelo de inspiración para los países centroamericanos durante la definición de su proceso de integración.

Hay, de hecho, unas similitudes entre modalidades institucionales, reglamentarias y organizativas y modos de hacer y pensar, narrativas y representaciones experimentadas en el proceso de la integración centroamericana y el proceso de integración de la Unión Europea.

Como se ha mencionado en el artículo, estas analogías son evidentes tanto a escala supranacional -por ejemplo, al considerar los procesos de definición del SICA y del Plan Trifinio- como a escala local donde la creación de mancomunidades, y en particular la Mancomunidad Trinacional Río Lempa, refleja el modelo de cooperación transfronteriza entre autoridades subestatales, originado en Europa en la década de 1950.

Además, desde el inicio, la Unión Europea ha desempeñado un papel directo y especialmente activo en el proceso de integración de la región del Trifinio. De hecho, la Comunidad Económica Europea financió los estudios preliminares para la elaboración del Plan Trifinio en la década de 1980 y aportó el 75 por ciento de los recursos económicos necesarios para la realización del Proyecto Piloto Trifinio durante la década de 1990. Asimismo, más recientemente, a través del programa Urb-Al III, la Unión ofrece un medio para el intercambio de mejores prácticas entre la Unión Europea y América Latina en materia de cooperación transfronteriza.

Sin embargo, como se ha mencionado, la transferencia de políticas (policy transfer) siempre implica una dinámica de apropiación y adaptación de las políticas a las características específicas locales. El caso del Trifinio confirma esta tendencia, ya que muestra una serie de diferencias con respecto a la experiencia europea y una serie de singularidades que lo caracterizan. Estas peculiaridades no están únicamente relacionadas con la fragilidad del sistema (que según una comparación con el europeo presenta, por ejemplo, un nivel más débil de integración y un contexto más frágil de descentralización y subsi- 
diariedad). En la región del Trifinio, en respuesta a los problemas locales específicos, se han desarrollado asimismo mecanismos específicos; por ejemplo, los relativos a la gestión de los fondos de la cooperación internacional o a la gestión de la gobernanza multinivel. En la región centroamericana, se ha venido realizando, por lo tanto, una verdadera apropiación de políticas inspiradas en el modelo europeo.

En conclusión, a nivel de América Latina, el Trifinio constituye un conjunto de lecciones aprendidas: políticas y prácticas transferidas desde la Unión Europea han experimentado un proceso de adaptación a las peculiaridades del contexto local. Hoy, el Trifinio, con sus rasgos y características propios, puede representar un ejemplo en el que otras iniciativas en América Latina puedan inspirarse, a fin de impulsar la difusión de políticas y prácticas de cooperación transfronteriza a nivel regional, a diferentes escalas. Por lo tanto, la región puede ser considerada como un laboratorio en el cual desarrollar un enfoque específico de cooperación transfronteriza, más compatible con las especificidades y las problemáticas locales.

\section{REFERENCIAS}

1. Artiaga, Raúl. 2003. El Trifinio, desafío y retos de una cuenca compartida. Consultado 1 de marzo de 2013 (http://seia.guanajuato.gob.mx/panel/document/phpver.php?Id=2075).

2. Benson, David y Andrew Jordan. 2011. "What Have We Learned from Policy Transfer Research? Dolowitz and Marsh Revisited." Political Studies Review 9 (2): 366-78.

3. Brenner, Neil. 2004. New State Spaces. Urban Governance and the Rescaling of Statehood. Oxford: Oxford University Press.

4. Caldentey, Pedro y José Romero. 2010. El SICA y la UE: la integración regional en una perspectiva comparada. Córdoba, España: Fundación ETEA para el Desarrollo y la Cooperación.

5. Cardona, Rokael. 2009. "La integración centroamericana desde lo local: la Mancomunidad Trinacional Fronteriza Río Lempa." Pp. 256-75 en Procesos de integración regional e internacionalización de los gobiernos locales, editado por Observatorio de Cooperación Descentralizada UE-AL, Anuario de la cooperación descentralizada. Montevideo: Observatorio de Cooperación Descentralizada UE-AL.

6. Celata, Filippo y Raffaella Coletti. 2011. "Le funzioni narrative dei confini nelle politiche di cooperazione transfrontaliera in Europa: una lettura interpretativa." Rivista Geografica Italiana 118 (2): 219-45. 
7. Coletti, Raffaella. 2010. "Cooperación transfronteriza y trayectorias de desarrollo: aprendizajes de la experiencia europea." Si Somos Americanos. Revista de Estudios Transfronterizos X (1): 161-80.

8. Comisión Trinacional del Plan Trifinio (CTPT). 1992. Plan Trifinio - El Salvador-Guatemala-Honduras. Washington: OEA.

9. ----. 1998. Tratado entre las Repúblicas de El Salvador, Guatemala y Honduras para la Ejecución del Plan Trifinio de 1997.

10. ----- 1999. Informe Anual 1998-Proyecto Piloto de Desarrollo de la Región del Trifinio. Proyecto ALA 88/14.

11. -----. 2004. Actualización del Plan Trifinio. Plan Trifinio. Una estrategia para el desarrollo de la Región.

12. Comunidad Europea. 1957. Tratado Constitutivo de la Comunidad Europea.

13. Conato, Dario. 2009. "Fronteras de tierra y de mar: de áreas conflictivas a espacios de colaboración e integración centroamericana." Pp. 103-38, en Cooperación transfronteriza e integración en América Latina: la experiencia del proyecto Fronteras Abiertas, editado por José Luis Rhi-Sausi y Dario Conato. Roma: CeSPI. Consultado 1 de marzo de 2013 (http://www.cespi.it/libro\%20Fronteras/00\%20FRONTERAS\%20light.pdf).

14. Dolowitz, David y David Marsh. 1996. "Who Learns What from Whom? A Review of the Policy Transfer Literature.” Political Studies 44 (2): 343-57.

15. Featherstone, Kevin y Claudio Radaelli. 2003. The politics of Europeanization. Oxford: Oxford University Press.

16. Guerrero, Orlando. 2004. "El proceso de integración centroamericana y la globalización, Anuario de derecho constitucional latinoamericano." Pp. 995-1010, en Anuario de Derecho Constitucional Latinoamericano, editado por Biblioteca Jurídica Virtual. México: Instituto de Investigaciones Jurídicas de la Universidad Nacional Autónoma de México. Consultado el 1 de marzo de 2013 (http://www.juridicas.unam.mx/publica/librev/rev/ dconstla/cont/2004.2/pr/pr27.pdf)

17. Hooghe, Liesbet y Gary Marks. 2001. Multi-Level Governance and European Integration. Boulder: Rowman \& Littlefield.

18. Mancomunidad Trinacional Fronteriza Río Lempa (MTFRL). 2008. Planificación Estratégica Territorial Trinacional 2008-2013. 
19. Marteles, Silvia. 2010. "Fortalecimiento de la gobernanza transfronteriza en América Latina a través de la cooperación descentralizada: la experiencia del programa Fronteras Abiertas." Si Somos Americanos. Revista de Estudios Transfronterizos X (1): 147-60.

20. McCann, Eugene y Kevin Ward. 2012. "Policy Assemblages, Mobilities and Mutations: Toward a Multidisciplinary Conversation.” Political Studies Review 10: 325-32.

21. Montero, Alfred y David Samuels (eds.). 2004. Decentralization and democracy in Latin America. Notre Dame, Indiana: University of Notre Dame Press.

22. Organización de los Estados Americanos (OEA) e Instituto Interamericano de Cooperación para la Agricultura (IICA). 1988. Plan de Desarrollo Regional Fronterizo Trinacional Trifinio (Plan Trifinio).

23. Olsen, Johan. 2002. “The Many Faces of Europeanization." Journal of Common Market Studies 40 (5): 921-52.

24. Peck, Jamie y Nik Theodore. 2001. "Exporting Workfare/Importing Welfare-to-Work: Exploring the Politics of Third Way Policy Transfer." Political Geography 20 (4): 427-60.

25. ----. 2010. "Mobilizing Policy: Models, Methods and Mutations." Geoforum 41 (2):16974 .

26. Perkmann, Markus. 2003. "Cross Border Regions in Europe. Significance and drivers of regional cross-border cooperation.” European Urban and Regional Studies 10 (2): 153-71.

27. Perkmann, Markus y Ngain-Ling Sum (eds.). 2002. Globalization, Regionalization and Cross Border Regions. Londres: Palgrave Macmillan.

28. Prince, Russell. 2010. "Policy Transfer as Policy Assemblage: Making Policy for the Creative Industries in New Zealand." Environment and Planning A 42 (1): 169-86.

29. Rhi-Sausi, José Luis y Dario Conato (coords.). 2009. Cooperación transfronteriza e integración en América Latina. Roma: CeSPI. Consultado el 1 de marzo de 2013. (http:// www.cespi.it/libro\%20Fronteras/00\%20FRONTERAS\%20light.pdf)

30. Rhi-Sausi, José Luis, Dario Conato, Nahuel Oddone y Danilo Salerno. 2011. Conceptualización de la cooperación fronteriza en el sistema de la integración centroamericana. Ideas y prácticas para la construcción de las microregiones de integración. Roma: CeSPI. Consultado el 1 de marzo de 2013. (http://www.cespi.it/PDF/CONCEPTUALIZACI\%C3\%93N\%20 \%20COOPERACI\%C3\%93N\%20FRONTERIZA.pdf) 
31. Rhi-Sausi, José Luis, Dario Conato y Nahuel Oddone. 2009. “Cooperación e integración transfronteriza en América Latina y el Mercosur." Pp. 11-31, en Cooperación transfronteriza e integración en América Latina: la experiencia del proyecto Fronteras Abiertas, editado por José Rhi Sausi y Dario Conato. Roma: CeSPI. Consultado 1 de marzo de 2013. (http://www.cespi.it/libro\%20Fronteras/01\%20FRONTERAS\%20light.pdf)

32. Sanna, Venere Stefania. 2011. Estudio comparado sobre la legislación e institucionalidad del ordenamiento y desarrollo territorial de los países que conforma el Sistema de la Integración Centroamericana (SICA) y la Unión Europea. Mancomunidad Trinacional Fronteriza Río Lempa y CeSPI, Roma. Consultado el 1 marzo de 2013 (http://www.cespi.it/PDF/ ESTUDIOpor ciento20COMPARADOpor ciento20ODTpor ciento20SICA-UE.pdf).

33. Schimmelfennig, Frank. 2010. "Europeanisation Beyond the Member States." Zeitschrift für Staats- und Europawissenschaften 8 (3): 319-39.

34. Task Team on South-South Cooperation (TT-SSC). 2011. Cooperación en la Región del Trifinio: un caso de cooperación transfronteriza Sur-Sur. Estudio de caso. Consultado el 1 de marzo de 2013 (www.southsouthcases.info/pdf/lac13.pdf)

35. Uitermark, Justus. 2005. "The genesis and evolution of urban policy: a confrontation of regulationist and governmentality approaches.” Political Geography 24: 137-63. 